\title{
La evaluación de la resolución
}

\section{de problemas en matemáticas:}

\section{concepciones y prácticas}

\section{de los profesores de secundaria}

\author{
Autora: Janeth A. Cárdenas Lizarazo • Autora de la reseña:Ana Caballero Carrasco \\ Tipo de tesis:Tesis doctoral \\ Directores: Lorenzo J. Blanco Nieto y Eloísa Guerrero Barona \\ Departamento: Didáctica de las Ciencias Experimentales y de las Matemáticas \\ Universidad: Universidad de Extremadura \\ Programa: Doctorado en Investigación en la Enseñanza y el Aprendizaje de las Ciencias Experimentales, Sociales y Matemáticas \\ Fecha de presentación: 22 de julio de 2014 \\ Fecha de recepción: junio 2015 • Fecha de aceptación: julio 2015 • Fecha de publicación: octubre 2015
}

Se extrae de esta tesis doctoral que la resolución de problemas de matemáticas (RPM) como contenido no solo ha sido considerada en la investigación en educación matemática por diferentes autores sino que así aparece también en los currículos de matemáticas. De esta forma, la RPM se convierte en objetivo de enseñanza y aprendizaje en matemáticas. La evaluación, entre sus múltiples funciones, se asume como el contraste entre los objetivos de enseñanza y los aprendizajes adquiridos por los estudiantes, por lo cual, la RPM debe ser, igualmente, un contenido que se ha de evaluar.

A la par, se deduce que con la evaluación es posible constatar, direccionar y apoyar los aprendizajes de los estudiantes. A través de la evaluación se reflejan las concepciones del profesor sobre el contenido y la evaluación, condicionando tanto la enseñanza, al enfatizar sobre cierto tipo de prácticas, como los aprendizajes de los estudiantes. Por tanto, indagar sobre las concepciones y prácticas de la evaluación de la RPM de los profesores de matemáticas, nos permite identificar aspectos relevantes sobre la evaluación en general y la evaluación de la RPM en particular.

En esta tesis se presentan como antecedentes una breve descripción de las demandas políticoeducativas sobre la evaluación de la RPM en Colombia. De la misma forma, se describen aspectos generales acerca de la evaluación, la RPM en el currículo y la evaluación de las matemáticas y de la RPM, resaltando los resultados descritos en diferentes investigaciones al respecto.

La investigación se centra en un estudio descriptivo-exploratorio en busca de conocer las prácticas y concepciones que desarrollan los profesores en torno a la evaluación de la RPM. Para ello, se trabaja con un grupo de profesores de matemáticas de secundaria de Bogotá (Colombia). Para la recogida y análisis de datos se opta por la complementación de metodologías cuantitativas y cualitativas, administrándose cuatro instrumentos de recogida de datos diferentes:

- Cuestionario sobre evaluación de la RPM. Se trata de un cuestionario de elaboración propia obtenido de un análisis de contenido de los lineamientos y estándares curriculares de matemáticas 
de Colombia. Los ítems son unidades de análisis referentes a la RPM como contenido. A través de este cuestionario se indaga acerca de lo que los profesores manifiestan «hacer» $\mathrm{y}$ "pensar» respecto a la evaluación de la RPM.

- Cuestionario sobre concepciones de los profesores acerca de la evaluación. Cuestionario adaptado a partir del de Brown y Remesal (2012), con el objeto de identificar las concepciones que mantienen los profesores de matemáticas acerca de la evaluación.

- Las actividades de evaluación empleadas por los profesores. Se trata de los instrumentos de evaluación aportados por los profesores de matemáticas y con los cuales afirman evaluar la RPM. Estos son analizados de manera cualitativa con una rúbrica específica y de elaboración propia. Se analizan en total 875 enunciados y 2.483 tareas propuestas por los docentes en las actividades de evaluación, identificando el tipo de demanda cognitiva que se exige al estudiante y el tipo de contexto, entre otros elementos que evidencian algunas prácticas evaluativas de los profesores de matemáticas.

- Entrevistas y discusiones de grupo a través de los cuales se profundiza y contrastan los resultados obtenidos a partir de los anteriores instrumentos.

Estos instrumentos son analizados de forma independiente y en combinación de forma que la triangulación de resultados permite caracterizar la evaluación de los profesores y comparar sus manifestaciones previas con su actividad en el aula.

Posteriormente se presenta la correspondiente discusión de resultados con autores que han encontrado resultados similares o cuestiones que se han evidenciado en otras investigaciones y que no se han encontrado en la presente.

Entre las conclusiones se señalan las dificultades y contradicciones que los profesores muestran ante la evaluación y su consideración de la RPM como contenido que ha de ser evaluado. Asimismo, se constata que la evaluación sigue pautas muy tradicionales y contradictorias con el currículo actual, con lo que los profesores manifiestan relevante en la enseñanza de las matemáticas y con las nuevas corrientes en educación matemática. A este respecto, se evidencia la aceptación de los profesores acerca de la ruptura entre el proceso de enseñanza/aprendizaje y la evaluación, basándose esta última, fundamentalmente, en su función certificativa.

Este estudio permite proponer a la administración educativa, a las instituciones de formación inicial y permanente del profesorado y a los profesores en ejercicio incidir en la formación del profesorado de matemáticas que permita visualizar y generar prácticas reales en las que pueda implementar una evaluación que posibilite un mayor acercamiento entre la teoría y la práctica de la evaluación en general y, más concretamente, de la evaluación de la RPM. 